hep-th/9609006

\title{
Understanding the Area Proposal for Extremal Black Hole Entropy
}

\author{
A. Ghosh 1 and P. Mitrał \\ Saha Institute of Nuclear Physics \\ Block AF, Bidhannagar \\ Calcutta 700 064, INDIA
}

\begin{abstract}
Whereas the usual understanding is that the entropy of only a non-extremal black hole is given by the area of the horizon, there are derivations of an area law for extremal black holes in some model calculations. It is explained here how such results can arise in an approach where one sums over topologies and imposes the extremality condition after quantization.
\end{abstract}

It has been known for quite some time now that a black hole can be assigned a temperature, which is a quantum effect, and is proportional to Planck's constant. Correspondingly, there is also an entropy [1, 2], with inverse dependence on Planck's constant and proportional to the area of the horizon. This entropy can be understood in a euclidean functional integral approach [3] where the integral is evaluated in the semiclassical approximation, i.e., replaced by the exponential of the negative of the minimum classical action, which is essentially a quarter of the area.

All this is about what are now called non-extremal black holes. One is now more often interested in a different class of black holes - the extremal ones. These are characterized by coinciding horizons and have qualitatively different features. Thus, the euclidean topology of an extremal black hole is different from that of the related non-extremal black holes. Again, the classical action of an extremal black hole vanishes. This results in an entropy which vanishes [1] or behaves like the mass [5], but certainly does not behave like the area.

\footnotetext{
${ }^{1}$ e-mail amit@tnp.saha.ernet.in

${ }^{2}$ e-mail mitra@tnp.saha.ernet.in
} 
Recently there have been some studies of black hole entropy in a string model which count states in what is believed to be a microscopic description of extremal black holes and come up with a quarter of the area. While the possibility of explicit counting is interesting, the result (see [6]) is intriguing in view of the earlier understanding that the area formula applies only to nonextremal black holes. It is true that the borderline between non-extremal and extremal cases is very thin and if one takes the extremal limit of non-extremal black holes instead of an extremal black hole directly, one obtains the area answer. But, as mentioned above, the euclidean topologies are different, so one should consider not the limit but the extreme black hole by itself; and then the string theorists' result does not match the thermodynamical answer. A simple way out of this mismatch would be to say that one of these calculations is wrong, but the fact that the area turns up in both the string calculation for the extremal case and in the thermodynamical calculation for the non-extremal case suggests that there is a deeper truth. Clearly, for some reason, a non-extremal case is appearing in the garb of an extremal case. How can this have happened? This is what we seek to understand.

Usually, when one quantizes a classical theory, one tries to preserve the classical topology. In this spirit, one usually seeks to have a quantum theory of extremal black holes based exclusively on extremal topologies. This leads to an entropy that vanishes or goes like the mass, as mentioned above. Clearly, since the string model gives a different answer, it does not work with the extremal topology, even implicitly. To simulate it, we shall try out a quantization where a sum over topologies is carried out. Thus, in our consideration of the functional integral, classical configurations corresponding to both topologies will be included. The extremality condition will be imposed not on the classical configurations but on the averages that result from the functional integration. We shall, following [3] and [7], use a grand canonical ensemble. Here the temperature and chemical potential are supposed to be specified as inputs, and the average mass $M$ and charge $Q$ of the black hole are outputs. So the actual definition of extremality that we have in mind for a Reissner- Nordström black hole is $Q=M$. This may be described as extremalization after quantization, as opposed to the usual approach of quantization after extremalization.

The action for the euclidean version of a Reissner - Nordström black hole 
on a four dimensional manifold $\mathcal{M}$ with a boundary is

$$
I=-\frac{1}{16 \pi} \int_{\mathcal{M}} d^{4} x \sqrt{g} R+\frac{1}{8 \pi} \int_{\partial \mathcal{M}} d^{3} x \sqrt{\gamma}\left(K-K^{0}\right)+\frac{1}{16 \pi} \int_{\mathcal{M}} d^{4} x \sqrt{g} F_{\mu \nu} F^{\mu \nu} .
$$

Here $\gamma$ is the induced metric on the boundary $\partial \mathcal{M}$ and $K$ the extrinsic curvature. A class of spherically symmetric metrics [7] is considered on $\mathcal{M}$ :

$$
d s^{2}=b^{2} d \tau^{2}+\alpha^{2} d y^{2}+r^{2} d \Omega^{2},
$$

with the variable $y$ ranging between 0 (the horizon) and 1 (the boundary), and $b, \alpha, r$ functions of $y$ only. There are boundary conditions as usual:

$$
2 \pi b(1)=\beta, r(1)=r_{B}, b(0)=0 .
$$

Here $\beta$ is the inverse temperature and $r_{B}$ the radius of the boundary which will be taken to infinity. There is another boundary condition involving $b^{\prime}(0)$ : It reflects the extremal/non-extremal nature of the black hole and is therefore different for the two conditions:

$$
\begin{aligned}
\frac{b^{\prime}(0)}{\alpha(0)} & =1 \ldots \text { in non }- \text { extremal case } \\
\text { but } & =0 \ldots \text { in extremal case. }
\end{aligned}
$$

The vector potential is taken to be zero and the scalar potential satisfies the boundary conditions

$$
A_{\tau}(0)=0, A_{\tau}(1)=\frac{\beta \Phi}{2 \pi i} .
$$

The variation of the action with this form of the metric and these boundary conditions leads to the Einstein - Maxwell equations. The solution of a subset of these equations, namely the Gauss law and the Hamiltonian constraint, is given by [7]

$$
\frac{r^{\prime}}{\alpha}=\left[1-\frac{2 m}{r}+\frac{q^{2}}{r^{2}}\right]^{1 / 2}, A_{\tau}^{\prime}=-\frac{i q b \alpha}{r^{2}}
$$

with the mass parameter $m$ and the charge $q$ arbitrary, except that $|q| \leq m$. The reason why these parameters have not been expressed as functions of $\beta, \Phi$ is that some of the equations have not been imposed. Instead of such 
imposition, the action may be simplified and then extremized with respect to $m, q \| 7$.

The value of the action corresponding to the solution depends on the boundary condition:

$$
\begin{aligned}
& I=\beta(m-q \Phi)-\pi\left(m+\sqrt{m^{2}-q^{2}}\right)^{2} \text { for non }- \text { extremal bc, } \\
& I=\beta(m-q \Phi) \text { for extremal bc. }
\end{aligned}
$$

The first line is taken from [7], where the non-extremal boundary condition was used in connection with a semiclassically quantized non-extremal black hole. The second line corresponds to the extremal boundary condition used in connection with a semiclassically quantized extremal black hole [8]. As the euclidean topologies of non-extremal and extremal black holes are different, quantization was done separately for the two cases in [7, 8]. The topology was selected before quantization.

As indicated above, a different approach is to be used here. The two topologies are to be summed over in the functional integral and the extremality condition imposed afterwards.

Thus the partition function is of the form

$$
\sum_{\text {topologies }} \int d \mu(m) \int d \mu(q) e^{-I(q, m)},
$$

with $I$ given by (7) as appropriate for non-extremal/extremal $q$.

The semiclassical approximation involves replacing the double integral by the maximum value of the integrand, i.e., by the exponential of the negative of the minimum $I$. We consider the variation of $I$ as $q, m$ vary in both topologies. It is clear from (7) that the non-extremal action is lower than the extremal one for each set of values of $q, m$. Consequently, the partition function is to be approximated by $e^{-I_{q, m}}$, where $I_{q, m}$ is the classical action for the non-extremal case, minimized with respect to $q, m$. The result, which should be a function of $\beta, \Phi$, can be read off [7]. It leads to an entropy equal to a quarter of the area for all values of $\beta, \Phi$. The averages $Q, M$, as opposed to the parameters $q, m$, are calculated from $\beta, \Phi$. We are interested in $|Q|=M$, i.e., the extremal black hole. This is obtained for limiting values

$$
\beta \rightarrow \infty, \quad|\Phi| \rightarrow 1, \text { with } \beta(1-|\Phi|) \text { finite }
$$


for the ensemble parameters and is described by the effective action

$$
I=\pi M^{2}=\frac{(\beta(1-|\Phi|))^{2}}{4 \pi} .
$$

It is worth emphasizing that for extremal black holes, the parameters $\beta, \Phi$ necessarily enter in the combination $\gamma \equiv \beta(1-|\Phi|)$ because the first law of thermodynamics takes the form

$$
d S=\frac{d M-\Phi d Q}{T}=\beta(1-|\Phi|) d M=\gamma d M .
$$

This combination does occur here as it also does in the case with purely extremal topology 8 .

Thus in the limit the partition function takes the form

$$
Z=e^{-\frac{\gamma^{2}}{4 \pi}}=e^{-\pi M^{2}}=e^{-\frac{A}{4}}
$$

This continues to correspond to an entropy of a quarter of the area of the horizon, which is the value of the entropy we sought to explain.

To reach this goal, we had to define extremality not by equating the classical parameters $q, m$ before quantization, but in terms of the averages $Q, M$ which are calculated from the ensemble characteristics $\beta, \Phi$ and which reduce to $q, m$ for the configuration with the minimum action in the semiclassical approximation. It is because of this altered definition, and the use of the sum over topologies, that non-extremal configurations have entered and we have obtained the area law for the entropy instead of the smaller values obtained in [4, 5]. This suggests that the string model result about the entropy implicitly involves a quantization procedure where the classical euclidean topology is ignored and the condition of extremality imposed only after quantization.

The derivation can be translated in terms of microscopic states. The relevant number of states can be split up into a number of states coming from the non-extremal sector and leading to the area formula and the small number of states coming from the extremal sector. The second contribution can be neglected in comparison to the first, and hence the area result survives.

It may be clarified here that we do not wish to suggest that this is the correct way of quantization. As in other areas of physics, there are different, often inequivalent, ways of quantization, all equally acceptable. The older results in quantum gravity literature correspond to quantization with fixed 
euclidean topology, while the recent string counting result agrees with, but does not explicitly involve, a sum over topologies.

It may be instructive to compare this approach with that of a recent paper [9] where the area law was found for the entropy of an extremal black hole. But in that work, the non-extremal topology was tacitly chosen without justification. As pointed out in [8], the area law does not appear if the approach of [7] is adapted to the extremal topology. The main point of the present work is to show how one can argue for the appearance of the nonextremal topology in the extremal case. Once the non-extremal topology appears, the emergence of the area answer is only natural.

Lastly, it should be pointed out that the functional integral is evaluated only in an approximation, but the variation of the action becomes sharp, and the approximation better, for large black holes. There will be corrections arising from fluctuations around the dominant configuration, which have been neglected as usual [3]. As the leading area term in the entropy has $\hbar$ in the

denominator, these corrections will be of order $\hbar^{0}$, but relatively small, unless the area itself is comparable to $\hbar$.

\section{References}

[1] J. Bekenstein, Phys. Rev. D7, 2333 (1973); Phys. Rev. D9, 3292 (1974)

[2] S. Hawking, Comm. Math. Phys. 43, 199 (1975)

[3] G. Gibbons and S. Hawking, Phys. Rev. D15, 2752 (1977)

[4] S. Hawking, G. Horowitz and S. Ross, Phys. Rev. D51, 4302 (1995)

[5] A. Ghosh and P. Mitra, Phys. Letters B357, 295 (1995); hep-th/9509090

[6] G. Horowitz, gr-qc/9604051

[7] H. W. Braden, J. D. Brown, B. F. Whiting and J. W. York, Phys. Rev. D42, 3376 (1990)

[8] A. Ghosh and P. Mitra, Phys. Rev. Letters, 77, 4848 (1996)

[9] O.B. Zaslavskii, Phys. Rev. Letters 76, 2211 (1996) 